\title{
Theoretical Limit in the Magnetization Reversal of Stoner Particles
}

\author{
X.R. Wang and Z.Z. Sun \\ Physics Department, The Hong Kong University of Science and Technology, Clear Water Bay, Hong Kong SAR, China
}

(Received 11 October 2006; published 12 February 2007)

\begin{abstract}
Magnetization reversal of uniaxial Stoner particles under the Slonczewski spin-transfer torques of polarized electric currents is investigated. Based on the modified Landau-Lifshitz-Gilbert equation of magnetization dynamics, the theoretical limit of critical currents required to reverse a magnetization with an arbitrary polarized current is obtained. Under a constant polarization degree and constant current amplitude, the optimal current pulse for the fastest magnetization reversal is derived. These results can be used as benchmarks to evaluate different reversal strategies besides other possible usages.
\end{abstract}

The advent of miniaturization and fabrication of magnetic particles at the nanometer scale [1] (called Stoner particles, where strong exchange interactions keep the magnetic moments of atoms rigid) makes the StonerWohlfarth (SW) problem [2] very relevant to nanosciences and nanotechnologies. While the magnetization dynamics under magnetic fields $[3,4]$ is well established, the manipulation of the magnetization dynamics by a spin-polarized electric current [5-10] is not thoroughly understood despite its importance in spintronics due to the low power consumption and the locality of currents. The spin-transfer torque from spin-polarized electric currents was independently suggested [5] by Slonczewski and Berger in 1996, and was verified by experiments [8]. Important issues are to lower critical currents required to reverse a magnetization [10] and to design a current pulse such that the magnetization can be switched from one state to another fast. Many reversal schemes $[9,10]$ have been proposed and examined. However, the theoretical limit of critical currents required to reverse a magnetization with an arbitrary polarized current is yet to be found. Consequently, other than comparing one reversal scheme with another, one has no objective criteria in evaluating an infinite number of possible reversal schemes. Neither one knows how much more critical currents could be lowered. The question of the optimal current pulse for the fastest magnetization reversal is also unknown. Finding these criteria forms the main topic of this Letter.

The itinerant electrons can mutually interact with local magnetic moments. Giant magnetoresistance is about the action of a magnetic moment on the electric currents, and the influence of the current on the magnetic moment is the subject of spin-transfer torque (STT). Consider a spinpolarized electric current entering into a Stoner particle with magnetization $\vec{M}$, the polarized electrons transfer their spin angular momenta to $\vec{M}$, resulting in STT [5]. Theoretical studies [5-7] show that the STT $\Gamma$ is proportional to the current with following form:

$$
\Gamma \equiv\left[\frac{d(\vec{M} V)}{d t}\right]_{\mathrm{STT}}=\frac{\gamma \hbar I}{\mu_{0} e} g(P, \vec{m} \cdot \hat{s}) \vec{m} \times(\vec{m} \times \hat{s}),
$$

where $\vec{m}, \hat{s}$ are the unit vector of $\vec{M}$ and polarization direction of the electric current, respectively. In the expression, $V, \hbar, \mu_{0}$, and $e$ denote the volume of the magnetic nanostructure, the Planck constant, the vacuum magnetic permeability, and the electron charge, respectively. $\gamma=$ $2.21 \times 10^{5}(\mathrm{rad} / \mathrm{s}) /(\mathrm{A} / \mathrm{m})$ is the gyromagnetic ratio. The exact microscopic formulation of the STT is still a subject of debate [6,7]. Different theories provide different expressions for function $g$ depending on the degree of the polarization $P$ of the current and relative angle between $\vec{m}$ and $\hat{s}$. Many experimental investigations [8] so far are consistent with the result of Slonczewski [5] which will be used throughout this study,

$$
g(P, \vec{m} \cdot \hat{s})=\frac{4 P^{3 / 2}}{(1+P)^{3}(3+\vec{m} \cdot \hat{s})-16 P^{3 / 2}} .
$$

The magnetization dynamics of $\vec{M}$ under an effective magnetic field $\vec{h}_{t}$ and a polarized current is governed by the modified Landau-Lifshitz-Gilbert (LLG) equation [6] with an additional term due to the STT of Eq. (1). The dimensionless form of the LLG equation is

$$
\left(1+\alpha^{2}\right) \frac{d \vec{m}}{d t}=-\vec{m} \times \vec{h}_{1}-\vec{m} \times\left(\vec{m} \times \vec{h}_{2}\right),
$$

where $\vec{h}_{1}=\vec{h}_{t}+\alpha a_{I} \hat{s}$, and $\vec{h}_{2}=\alpha \vec{h}_{t}-a_{I} \hat{s} . t$ in Eq. (3) is in the units of $(\gamma M)^{-1} \cdot a_{I}=\hbar I g /\left(\mu_{0} e M^{2} V\right)$ is a dimensionless parameter, and $\alpha$ is the Gilbert damping constant. Both magnetization and magnetic field are in the units of $M$. The total field $\vec{h}_{t}=\vec{h}+\vec{h}_{i}$ includes both the applied magnetic field $\vec{h}$ and the internal field $\vec{h}_{i}$ due to the magnetic anisotropic energy density $w(\vec{m}), \vec{h}_{i}=$ $-\nabla_{\vec{m}} w(\vec{m}) / \mu_{0} . \vec{h}_{1}$ and $\vec{h}_{2}$ are in general noncollinear, thus the dynamics with the additional STT term in Eq. (3) is very different from that without this term which describes a Stoner particle in a magnetic field only. The particle energy can only decrease in a static magnetic field since the field cannot be an energy source [4]. However, a polarized electric current can pump energy into the magnet, resulting in very rich physics [6]. According to Eq. (3), $\vec{m}$ undergoes a precessional motion around field $\vec{h}_{1}$ and a 
damping motion toward a different field $\vec{h}_{2}$. Equation (3) is a first order of differential equation, and there is no concept of inertial in terms of magnetization velocity. The magnetization obtains its velocity instantaneously from STT or fields (infinite acceleration).

Equation (3) is the starting point of most recent theoretical and experimental works. $\vec{m}$ can be conveniently described by the polar angle $\theta$ and the azimuthal angle $\phi$ in the spherical coordinate (Fig. 1). The problem of using an STT alone $(\vec{h}=0)$ to reverse a magnetization is as follows: Consider a magnetic nanoparticle with a given Gilbert damping constant $\alpha$ and the anisotropy $w(\vec{m})$. In the absence of a polarized current, $\vec{m}$ has two stable directions, $\theta=0$ and $\theta=\pi$, along its easy axis ( $z$ axis). The goal is to reverse the initial state (say $\theta=0$ ) to the target state, $\theta=\pi$, fast by using a proper polarized electric current pulse. Obviously, there are an infinite number of paths that connect $\theta=0$ to $\theta=\pi$. L1 and L2 in Fig. 1 are two examples. Each of these paths can be used as a magnetization reversal route (path). For a given reversal route, there are an infinite number of polarized current pulses that can reverse the magnetization. An interesting question is whether the best reversal path exists so that the reversal time is the shortest for a constant current amplitude $I$ and a constant degree of polarization $P$ of the current. Another issue is what is the smallest polarized current (theoretical limit of critical currents) out of all possible reversal current pulses. $\vec{m}$ satisfies nonlinear equation Eq. (3) whose solution for an arbitrary time-dependent current is in general difficult to find. However, its inverse problem is simple. Namely, for a given reversal process, the corresponding required polarized current can be trivially obtained from Eq. (3). Thus, answers to the above two questions can be found. To show how one can obtain these answers, we consider uniaxial particles of magnetic anisotropy $w=w\left(m_{z}\right)$ with its easy axis along the $z$ direction so that $\vec{h}_{i} \equiv f(\cos \theta) \hat{z}$. Let $\hat{e}_{r}, \hat{e}_{\theta}, \hat{e}_{\phi}$ be the three spherical unit vectors of $\vec{m}$, so $\vec{h}_{i}=-f \sin \theta \hat{e}_{\theta}+f \cos \theta \hat{e}_{r}$. In terms of $\theta$ and $\phi$, Eq. (3) can be written as

$$
\begin{aligned}
\left(1+\alpha^{2}\right) \dot{\theta} & =a_{I}\left(\alpha s_{\phi}-s_{\theta}\right)-\alpha f(\cos \theta) \sin \theta, \\
\left(1+\alpha^{2}\right) \sin \theta \dot{\phi} & =-a_{I}\left(\alpha s_{\theta}+s_{\phi}\right)+f(\cos \theta) \sin \theta .
\end{aligned}
$$

Here $s_{\theta}, s_{\phi}, s_{r}$ are the $\hat{e}_{\theta}, \hat{e}_{\phi}$, and $\hat{e}_{r}$ components of $\hat{s}$.

It is important to define rigorously and precisely critical currents and the theoretical limit of critical currents first. Assume $C^{L, q}(t) \hat{s}(t)$ to be the polarized electric current pulse of design $q$ along magnetization reversal route $L$ and $C^{L, q}(t)$ to be the current amplitude, three interesting quantities can be defined as follows:

Switching current $I^{L, q}$. - The switching current $I^{L, q}$ of design $q$ along route $L$ is defined to be the maximum of $\left|C^{L, q}(t)\right|$ for all $t, I^{L, q}=\max \left\{\left|C^{L, q}(t)\right| ; \forall t\right\}$.

Critical current $I^{L}$ of reversal route $L$. - The critical current along route $L$ is defined to be the smallest value of

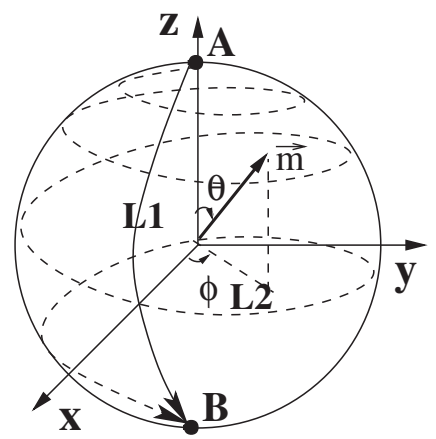

FIG. 1. Points A and B represent the initial and the target states, respectively. The solid curve L1 and dashed curve L2 illustrate two possible reversal routes.

$I^{L, q}$ for all possible designs $q$ that will force the magnetization to move along $L$, i.e., $I^{L}=\min \left\{I^{L, q} ; \forall q\right\}$.

Theoretical limit of critical currents $I_{c}$. - The critical current limit $I_{c}$ is defined as the smallest value of $I^{L}$ out of all possible routes, i.e., $I_{c}=\min \left\{I^{L} ; \forall L\right\}$. With these definitions, we present our results and proofs.

Claim 1.-Under the constraint of the constant current magnitude and constant polarization degree $P$, the optimal time-dependent polarization direction of the current is

$$
\begin{aligned}
s_{r}^{*} & =\frac{(1+P)^{3}}{16 P^{3 / 2}-3(1+P)^{3}}, \\
s_{\phi}^{*} & =-\alpha s_{\theta}^{*}=\frac{\alpha}{\sqrt{1+\alpha^{2}}} \sqrt{1-s_{r}^{* 2},}
\end{aligned}
$$

such that the reversal time from $\theta=0$ to $\theta=\pi$ is the shortest.

Proof.-According to Eq. (4), different $\hat{s}(t)$ generates different angular velocities for $\theta$ and $\phi$. Thus, the magnetization reversal time from $\theta=0$ to $\theta=\pi$ is given by $T \equiv$ $\int_{0}^{\pi} d \theta / \dot{\theta}$. To find the optimal $\hat{s}(t)$ that minimizes $T$, one only needs $a_{I}\left(\alpha s_{\phi}-s_{\theta}\right)$ or $g\left(P, s_{r}\right)\left(\alpha s_{\phi}-s_{\theta}\right)$ to be maximum such that $\dot{\theta}$, according to Eq. (4), will be the largest at any $\theta$. This observation is important and it can be applied to other function forms of $g$. Because $s_{r}^{2}+s_{\theta}^{2}+s_{\phi}^{2}=1$, the maximum of $g\left(P, s_{r}\right)\left(\alpha s_{\phi}-s_{\theta}\right)$ can be obtained from the standard Lagrange multiplier method in which one introduces $F \equiv g\left(P, s_{r}\right)\left(\alpha s_{\phi}-s_{\theta}\right)-\lambda\left(s_{r}^{2}+s_{\theta}^{2}+s_{\phi}^{2}\right)$. By setting the partial derivatives of $F$ with respect to $s_{i}$ $(i=r, \theta, \phi)$ to zeros, the maximum of $g\left(P, s_{r}\right)\left(\alpha s_{\phi}-s_{\theta}\right)$ is

$$
\left[g\left(P, s_{r}\right)\left(\alpha s_{\phi}-s_{\theta}\right)\right]_{\max }=\sqrt{1+\alpha^{2}} G(P),
$$

where

$$
G(P)=g\left(P, s_{r}^{*}\right) \sqrt{1-s_{r}^{* 2}}
$$

and the optimal $\hat{s}^{*}$ is given by Eq. (5).

Claim 2. - The theoretical limit of critical currents is

$$
I_{c}=\frac{\mu_{0} e M^{2} V}{\hbar G(P)} \frac{\alpha}{\sqrt{1+\alpha^{2}}} Q .
$$


Here $Q \equiv \max \{f(\cos \theta) \sin \theta\}$ for $\theta \in[0, \pi]$, and $G(P)$ is given by Eqs. (5) and (7).

Proof.-Under the optimal design of Eq. (5), $\theta(t)$ and $\phi(t)$ satisfy, respectively,

$$
\dot{\theta}=\frac{\hbar I}{\mu_{0} e M^{2} V} \frac{G(P)}{\sqrt{1+\alpha^{2}}}-\alpha f(\cos \theta) \sin \theta /\left(1+\alpha^{2}\right)
$$

and

$$
\dot{\phi}=f(\cos \theta) /\left(1+\alpha^{2}\right) .
$$

For a uniaxial model, the critical current limit $I_{c}$ can be obtained by setting the smallest value of $\dot{\theta}$ [Eq. (9)] to 0 in an optimal pulse. This is because $\dot{\theta}$ cannot be negative if the magnetization of a uniaxial particle moves from $\theta=0$ to $\theta=\pi$. Therefore, the first term in Eq. (9) must exceed the second term due to magnetic anisotropy for all $\theta$ 's ( $\in$ $[0, \pi])$ in a reversal. This simplicity for a uniaxial model comes from the $\phi$ independence of Eq. (9). Since Eq. (9) is the largest velocity for an arbitrary $\theta$ under the best choice of the polarization direction of a current, the critical current limit should be the one when the smallest $\dot{\theta}$ (for all $\theta$ ) is zero. Thus the critical current limit is Eq. (8). $\quad$ Q.E.D.

It is proper to make a few remarks here. (i) According to Eq. (5), $s_{r}^{*}=-1$ and $s_{\theta}^{*}=s_{\phi}^{*}=0$ when $P=1$. Then it seems that $\dot{\theta}=0$ at $\theta=0$ and $\pi$ according to Eq. (4). But this is not correct since $a_{I}$ diverges at $s_{r}=-1$ for $P=1$. In fact, $\dot{\theta}$ diverges under the limit of $s_{r} \rightarrow-1$ at $P=1$. This peculiar feature of Slonczewski's formula [Eq. (2)] may be an artifact which is a subject of debate [7]. (ii) An optimal pulse amount is used to switch magnetization of a nanomagnet by keeping the polarization of the current fixed with respect to the magnetization being switched. (iii) Although the optimal $\hat{s}^{*}$ appears to depend only on damping constant $\alpha$ and $P$, not on $f(\cos \theta)$, it is in fact time dependent because $\hat{s}$ is expressed in a moving frame whose coordinate units $\hat{e}_{r}, \hat{e}_{\theta}, \hat{e}_{\phi}$ vary with the time. (iv) From Eqs. (9) and (10), the optimal reversal route is given by

$$
\frac{d \theta}{d \phi}=\frac{\alpha Q}{f(\cos \theta)}\left[I / I_{c}-f(\cos \theta) \sin \theta / Q\right] .
$$

Thus, a solution passing through $\theta=0$ and $\pi$ simultaneously exists when and only when $I>I_{c}$. For $I<I_{c}$, the system undergoes a stable precession motion [6] [ $\theta$ satisfies $I / I_{c}=f(\cos \theta) \sin \theta / Q$ ] even under a pulse of Eq. (5). The evolution of $\vec{m}$ under the optimal polarization pulse (meaningful only for $I>I_{c}$ ) can be found by simply solving $\dot{\theta}=\alpha Q /\left(1+\alpha^{2}\right)\left[I / I_{c}-f(\cos \theta) \sin \theta / Q\right]$ and $\dot{\phi}=$ $f(\cos \theta) /\left(1+\alpha^{2}\right)$. It is clear that magnetic anisotropy $f(\cos \theta)$ shall influence the evolution of $\vec{m}$ which in turn influences the time dependence of $\hat{s}^{*}$. Thus, if they were to change $f(\cos \theta)$ and nothing else, the time-dependent $\hat{s}^{*}$ would be different.

One notices that the derivation of $I_{c}$ [Eq. (8)] does not require constant $I$ and $P$. The result should be the same even for a time-dependent $I$ and $P$ as long as STT is proportional to $I$ and $g$. To see how far that the best value of critical currents in the most advanced strategies [10] with the fixed current polarization direction is from the theoretical limit, let us compare it with the critical current limit of Eq. (8) for $w(\vec{m})=-k m_{z}^{2} / 2$. For this magnetic anisotropy, the theoretical limit of critical currents is $I_{c}=$ $\left[\mu_{0} e M^{2} V / 2 \hbar G(P)\right]\left[\alpha / \sqrt{1+\alpha^{2}}\right] k$. It is known [10] that the critical current for the polarization direction $\hat{s}$ parallel to the easy axis of $\vec{m}$ (parallel configuration) is smaller than that when $\hat{s}$ is perpendicular to the easy axis (perpendicular configuration). The critical current in the parallel configuration for the same magnetic anisotropy is $[5,10]$

$$
I_{c}^{\|}=\frac{\mu_{0} e M^{2} V}{\hbar g(P, 1)} \alpha k \text {. }
$$

Figure 2 is the plot of $I_{c}$ versus damping constant $\alpha$ for $P=0.4$ which is a realistic value for magnetic materials. The dashed line is of Eq. (11), and the solid line is the theoretical limit of the critical current which saturates to a constant at large $\alpha$ limit. At $\alpha=0.1$, critical current limit $I_{c}$ is about one fourth of that given by Eq. (11), showing a large room for the improvement. The difference between Eqs. (8) and (11) depends on the degree of polarization $P$. Figure 3 is $I_{c}$ vs $P$ at $\alpha=0.1$. It should be pointed out that zero $I_{c}$ in Eq. (8) at $P=1$ is an artificial result originated from the divergence of $g(1, x)$ at $x=-1$ in the Slonczewski's theory [5]. This divergence is removed in other formulations of $g$ [7].

For $w(\vec{m})=-k m_{z}^{2} / 2$, it is straightforward to integrate Eq. (9), and obtain the reversal time $T$ from $\theta=0$ to $\theta=$ $\pi\left[I_{c}\right.$ is given in Eq. (8)],

$$
T=\frac{2}{k} \frac{\left(\alpha^{2}+1\right) \pi}{\alpha \sqrt{\left(I / I_{c}\right)^{2}-1}} \quad\left(I>I_{c}\right) .
$$

In the weak damping limit $(\alpha \rightarrow 0)$ or large current limit $(I \rightarrow \infty), T \propto \pi / I$. To have an idea about the order of magnitudes for the critical current limit and the time scale considered here in a realistic setup, let us consider a Co nanostructure [10] of $3 \mathrm{~nm}$ thick and lateral dimension of $30 \times 60(\mathrm{~nm})^{2}$. Other parameters [10] are $\alpha=0.01$,

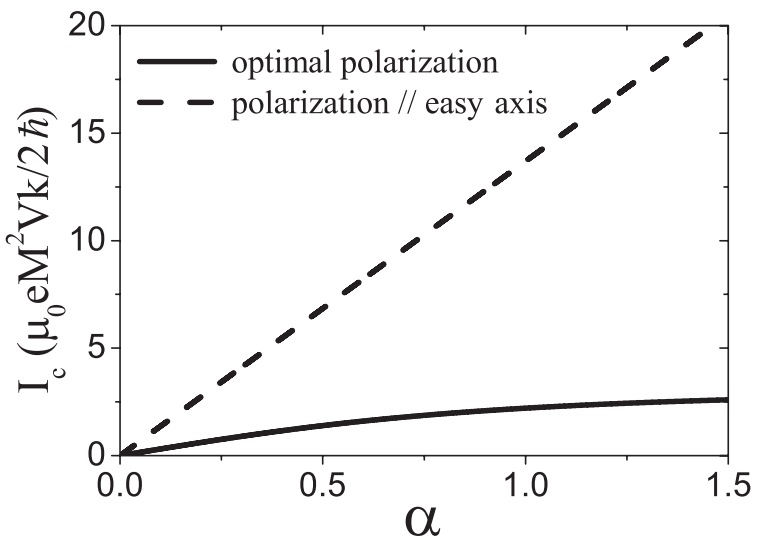

FIG. 2. $I_{c}$ vs $\alpha$ for $P=0.4$ and a uniaxial model of $w(\vec{m})=$ $-k m_{z}^{2} / 2$. 


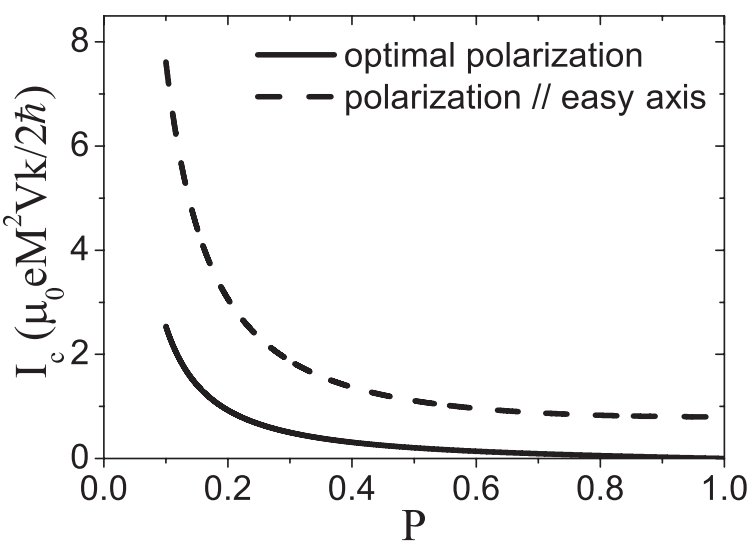

FIG. 3. $I_{c}$ vs $P$ at $\alpha=0.1$. The rest of the system parameters are the same as that of Fig. 2.

$M=1.4 \times 10^{6} \mathrm{~A} / \mathrm{m}, k=7 \times 10^{4} \mathrm{~J} / \mathrm{m}^{3}$, and $P=0.4$. Then, the theoretical limit of critical currents is about $I_{c} \simeq$ $18 \mu \mathrm{A}$ [current density of about $10^{6} \mathrm{~A} /(\mathrm{cm})^{2}$ ] compared with $80 \mu \mathrm{A}$ from Eq. (11). The optimal switching time is about $360 \mathrm{ps}$ for $I=1.8 \mathrm{~mA}>I_{c}$ [current density of $\left.10^{8} \mathrm{~A} /(\mathrm{cm})^{2}\right]$. Using parameters from Ref. [11], the theoretical limit is about $I_{c}=1 \mathrm{~mA}$ against an experimental value of about $8 \mathrm{~mA}$ [11]. The switching time from Eq. (12) is about $0.001 \mu \mathrm{s}$ at $I=3 \mathrm{~mA}$ which should be compared with an experimental switching time of $0.1-1 \mu \mathrm{s}$ at $I=8 \mathrm{~mA}$. This comparison shows that there is much room to be improved in experiment.

Although our results are obtained for uniaxial Stoner particles with a specific function of $g$, the basic ideas and approaches can in principle be generalized to the nonuniaxial cases with other $g$ functions. The results are also applicable to the case of nonzero static external magnetic field since one could add $\vec{h}$ to $\vec{h}_{i}$ by modifying the anisotropy energy density. To lower critical currents to its theoretical limit, one needs to find a way to generate an arbitrary spin-polarized electric current. This is an experimental challenge which may be realizable by using the socalled "spin-flip transistor" [12]. Another possible way to generate a desired current is through controlling the motion of the magnetization of the thick magnet in the conventional double sandwiched magnetic pillar [10]. The significance of our results is, however, that they provide benchmarks in evaluating a strategy so that we know how far the critical current in the strategy is from the theoretical limit, and how fast one may further increase in the magnetization reversal for a given electric power. From the results, one can also understand why any breakthrough in the spin-polarized current generation shall lead to the great leap forward in magnetization manipulation. It should also be pointed out that a real magnetization reversal is always done at a finite temperature. Strictly speaking, all quantities defined here at the finite temperature are meaningful only in the statistical sense. In general, thermal agitation can lower the critical switching field and current by thermally activating the system to cross the potential barrier [9].

In conclusion, we found the theoretical limit of critical currents which is far below the lowest critical current known today, showing a large room for future improvement. For a constant current amplitude and its polarization degree, an optimal time-dependent current polarization is obtained such that the magnetization reversal time is the shortest.

This work is supported by UGC, Hong Kong, through RGC CERG Grant No. 603106.

[1] M. H. Pan, H. Liu, J. Z. Wang, J. F. Jia, Q. K. Xue, J. L. Li, S. Qin, U. M. Mirdaidov, X. R. Wang, J. T. Market, Z. Y. Zhang, and C. K. Shih, Nano Lett. 5, 87 (2005).

[2] Spin Dynamics in Confined Magnetic Structures I \& II, edited by B. Hillebrands and K. Ounadjela (SpringerVerlag, Berlin, 2001).

[3] L. He and W.D. Doyle, IEEE Trans. Magn. 30, 4086 (1994); Y. Acremann, C. H. Back, M. Buess, O. Portmann, A. Vaterlaus, D. Pescia, and H. Melchior, Science 290, 492 (2000); Z. Z. Sun and X. R. Wang, Phys. Rev. B 71, 174430 (2005).

[4] Z.Z. Sun and X.R. Wang, Phys. Rev. B 73, 092416 (2006); 74, 132401 (2006); Phys. Rev. Lett. 97, 077205 (2006).

[5] J. Slonczewski, J. Magn. Magn. Mater. 159, L1 (1996); L. Berger, Phys. Rev. B 54, 9353 (1996).

[6] J. Z. Sun, Phys. Rev. B 62, 570 (2000); Z. Li and S. Zhang, ibid. 68, 024404 (2003); Y. B. Bazaliy, B. A. Jones, and S. C. Zhang, ibid. 57, R3213 (1998); 69, 094421 (2004).

[7] A. Brataas, Y. V. Nazarov, and G. E. W. Bauer, Phys. Rev. Lett. 84, 2481 (2000); X. Waintal, E. B. Myers, P.W. Brouwer, and D.C. Ralph, Phys. Rev. B 62, 12317 (2000); M. D. Stiles and A. Zangwill, ibid. 66, 014407 (2002).

[8] M. Tsoi, A. G. M. Jansen, J. Bass, W.-C. Chiang, M. Seck, V. Tsoi, and P. Wyder, Phys. Rev. Lett. 80, 4281 (1998); E. B. Myers, D. C. Ralph, J. A. Katine, R. N. Louie, and R. A. Buhrman, Science 285, 867 (1999); J. A. Katine, F. J. Albert, R. A. Buhrman, E. B. Myers, and D.C. Ralph, Phys. Rev. Lett. 84, 3149 (2000).

[9] Z. Li and S. Zhang, Phys. Rev. B 69, 134416 (2004); W. Wetzels, G.E.W. Bauer, and O.N. Jouravlev, Phys. Rev. Lett. 96, 127203 (2006).

[10] J. Sun, J. Magn. Magn. Mater. 202, 157 (1999); Nature (London) 425, 359 (2003); K. J. Lee, O. Redon, and B. Dieny, Appl. Phys. Lett. 86, 022505 (2005); J. Manschot, A. Brataas, and G.E.W. Bauer, ibid. 85, 3250 (2004); A. D. Kent, B. Zyilmaz, and E. del Barco, ibid. 84, 3897 (2004).

[11] R. H. Koch, J. A. Katine, and J. Z. Sun, Phys. Rev. Lett. 92, 088302 (2004).

[12] X. Wang, G. E. W. Bauer, and A. Hoffmann, Phys. Rev. B 73, 054436 (2006). 